\title{
Mixed infections with Chlamydia and porcine epidemic diarrhea virus - a new in vitro model of chlamydial persistence
}

\author{
Nicole Borel ${ }^{1 *}$, Claudia Dumrese ${ }^{2}$, Urs Ziegler ${ }^{2}$, Andrea Schifferli ${ }^{1}$, Carmen Kaiser ${ }^{1}$, Andreas Pospischil ${ }^{1}$
}

\begin{abstract}
Background: Chlamydiae induce persistent infections, which have been associated with a wide range of chronic diseases in humans and animals. Mixed infections with Chlamydia and porcine epidemic diarrhea virus (PEDV) may result in generation of persistent chlamydial infections. To test this hypothesis, an in vitro model of dual infection with cell culture-adapted PEDV and Chlamydia abortus or Chlamydia pecorum in Vero cells was established.

Results: Infected cultures were investigated by immunofluorescence (IF), transmission electron microscopy (TEM) and re-infection experiments. By IF, Chlamydia-infected cells showed normal inclusions after 39 hpi. Dual infections with Chlamydia abortus revealed a heterogenous mix of inclusion types including small inclusions consisting of aberrant bodies (ABs), medium-sized inclusions consisting of ABs and reticulate bodies and normal inclusions. Only aberrant inclusions were observable in dual infection experiments with Chlamydia pecorum and PEDV. TEM examinations of mixed infections with Chlamydia abortus and Chlamydia pecorum revealed aberrant chlamydial inclusions containing reticulate-like, pleomorphic ABs, which were up to $2 \mu \mathrm{m}$ in diameter. No re-differentiation into elementary bodies (EBs) was detected. In re-infection experiments, co-infected cells produced fewer EBs than monoinfected cells.

Conclusions: In the present study we confirm that PEDV co-infection alters the developmental cycle of member species of the family Chlamydiaceae, in a similar manner to other well-described persistence induction methods. Interestingly, this effect appears to be partially species-specific as Chlamydia pecorum appears more sensitive to PEDV co-infection than Chlamydia abortus, as evidenced by TEM and IF observations of a homogenous population of aberrant inclusions in PEDV - Chlamydia pecorum co-infections.
\end{abstract}

\section{Background}

Chlamydiae are implicated in a wide variety of diseases in both animals and humans. Although acute infections in animal chlamydioses are the most commonly reported, chronic chlamydial infections are also associated with a variety of diseases in humans and animals. These latter infections are characterized by inflammation and scarring resulting in significant damage of the host. A causative role in chronic diseases requires that chlamydiae persist within infected tissue for extended periods of time. Current theories, based primarily on in vitro data, suggest that chlamydial persistence, and the resulting chronic inflammation, is linked to

\footnotetext{
* Correspondence: n.borel@access.uzh.ch

'Institute of Veterinary Pathology, Vetsuisse Faculty, University of Zurich, Zurich, Switzerland
}

morphological and metabolic conversion of the actively replicating and intracellular reticulate body (RB) into an alternative, non-replicative form known as an aberrant body $(\mathrm{AB})[1]$. In vitro, alterations of the normal developmental cycle of Chlamydia trachomatis and Chlamydia pneumoniae can be induced by Interferon- $\gamma$ (IFN- $\gamma$ ), tumor necrosis factor- $\alpha$ (TNF- $\alpha$ ) and penicillin G exposure as well as amino acid or iron deprivation and monocyte infection $[2,3]$. To date, in vitro models for animal pathogens, Chlamydia abortus and Chlamydia pecorum have not been described although both organisms are associated with chronic disease in koalas and small ruminants [1].

In pigs, several chlamydial species, including Chlamydia abortus, Chlamydia psittaci, Chlamydia pecorum and Chlamydia suis, have been implicated in a variety of 
disease conditions including conjunctivitis, pneumonia, pericarditis, polyserositis, arthritis, abortion and infertility [4]. In the gastrointestinal tract, chlamydiae appear to be highly prevalent but only occasionally cause enteritis. They have been found in the intestine of diarrheic and healthy pigs and could be demonstrated in mixed enteric infections [5-7]. Pospischil and Wood [7] first described an association between Chlamydiaceae and lesions in the intestinal tract of pigs and assumed a synergistic effect in co-existence with Salmonella typhimurium. Further, mixed infections with Eimeria scabra, cryptosporidia, and porcine epidemic diarrhea virus (PEDV) have been described in the past. PEDV, a member of the family Coronaviridae, is a well-known cause of diarrhea in pigs. After the identification of PEDV in 1978 by Pensaert and Debouck [8], more than a decade passed before the virus could be adapted for propagation in cell cultures. Examination of infected Vero cell cultures by direct immunofluorescence revealed single cells with granular cytoplasmic fluorescence as well as formation of syncytia with up to 50-100 nuclei or more. Typical features of syncytial cells were growth, fusion and detachment from cell layers after they had reached a certain size [9]. Biomolecular studies revealed major genomic differences between cell culture-adapted (ca)PEDV and wild type virus $[10,11]$.

Cell culture model of co-infection with ca-PEDV and Chlamydia has been established recently [12] to investigate the interaction of ca-PEDV and Chlamydiaceae in mixed infections and to detect possible synergistic or additive effects of possible significance in clinical enteric disease in pigs. In that study, abnormally large chlamydial forms were observed in dually infected cell layers by immunofluorescence suggesting that ca-PEDV co-infection might alter the chlamydial developmental cycle in a manner similar to that observed during persistent infections. To confirm these initial observations, we established a cell culture model of mixed infections with Chlamydia and a cell culture-adapted porcine epidemic diarrhea virus (ca-PEDV) and hypothesized that this would result in the generation of persistent chlamydial forms. This data demonstrates that ca-PEDV co-infection, indeed, alters the developmental cycle of Chlamydia pecorum and Chlamydia abortus in a similar manner to other inducers of chlamydial persistence.

\section{Results}

Vero cells can be co-infected with Chlamydia and ca-PEDV Immunofluorescence (IF) labeling was used to investigate the morphologic differences of Chlamydia between monoinfected and dually infected monolayers using Chlamydia and ca-PEDV specific antibodies. Control and mock-infected cells did not stain with either antibody.
Ca-PEDV monoinfected cells showed brilliant and distinct, red cytoplasmic fluorescence. Syncytia were characterized by accumulation of nuclei in the center or the periphery of the multi-nucleated cells and moderate to bright, fine-granular, cytoplasmic ca-PEDV labeling (Figure 1b). Syncytia were categorized into small (2-15 nuclei), medium (16-30 nuclei) and large (more than 30 nuclei). In single infection experiments, syncytia at $24 \mathrm{~h}$ post infection were mostly large with fewer medium sized syncytia observed (data not shown). Numbers of syncytia in ca-PEDV single and dual infections were counted on the whole coverslip and mean values were determined. No difference of viral syncytia numbers for ca-PEDV monoinfection and dual infection with Chlamydia abortus were seen (data not shown). In contrast, numbers of viral synyctia in dual infections with Chlamydia pecorum were diminished compared to the respective ca-PEDV single infections (Table 1).

IF microscopy of chlamydial single infections revealed intracytoplasmic, mainly round to ovoid, sharply outlined inclusions with brilliant, green fluorescence. Chlamydia abortus and Chlamydia pecorum infected cells had one to five, finely granular (consisting mainly of EBs) inclusion(s) per cell at $39 \mathrm{~h}$ post infection (Figure

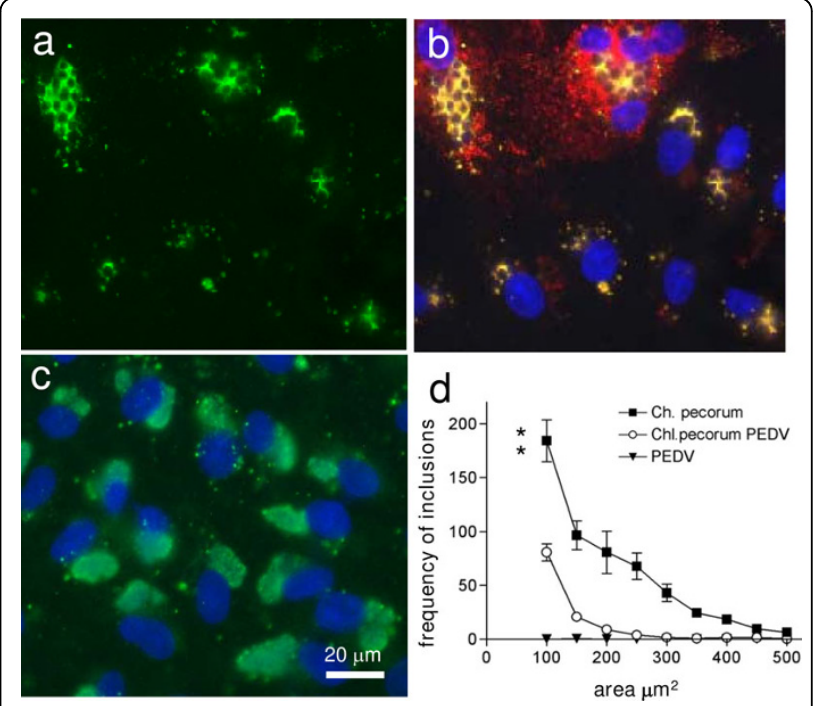

Figure 1 Morphology of Chlamydia pecorum mono- and coinfection with PEDV. a) Vero cells were infected with Chlamydia pecorum $1 \mathrm{MOI}$ for $39 \mathrm{~h}$, with subsequent PEDV inoculation and labelled with an anti-Chlamydia antibody (green); b) double infected monolayer were labelled for ca-PEDV in red, Chlamydia in green and DNA in blue; c) Chlamydia pecorum mono-infected Vero cells labelled with an anti-Chlamydia antibody (green) and DNA staining (blue); d) Inclusion size was measured as described and the frequency of chlamydial inclusions assembled into sizes of $50 \mu^{2}$ area groups depicted. The difference between mono and double infected monolayers was statistically analyzed using students t-test. The groups were significantly different with $p=0.0044$. 
Table 1 Numbers of syncytia for ca-PEDV monoinfection compared with dual infection with Chlamydia pecorum

\begin{tabular}{llll}
\hline \multicolumn{4}{c}{ Number of syncytia $^{a}$} \\
\hline Experimental group $^{\boldsymbol{b}}$ & Experiment & Experiment $^{\text {Experiment }}$ \\
& $\mathbf{1}$ & $\mathbf{2}$ & $\mathbf{3}$ \\
\hline Mock & 0 & 0 & 0 \\
\hline Ca-PEDV & 102 & 23 & 159 \\
\hline Chlamydia pecorum & 0 & 0 & 0 \\
\hline Chlamydia pecorum/ca- & 8 & 3 & 16 \\
PEDV & & &
\end{tabular}

${ }^{a}$ Numbers of syncytia for ca-PEDV monoinfection and dual infection with Chlamydia pecorum were counted on the whole coverslip.

${ }^{b}$ Vero cells were mock infected (Mock), C. pecorum infected, ca-PEDV infected (ca-PEDV) and Chlamydia pecorum/ca-PEDV co-infected as described.

1c \&2a). In general, chlamydial inclusions were smaller and had more variable forms in Chlamydia pecorum than in Chlamydia abortus single infections. Infectivity was almost $100 \%$ and a moderate number of free EBs could be observed.

\section{Ca-PEDV co-infection alters morphology and size of}

\section{chlamydial inclusions}

Compared to single infections, the size and shape of chlamydial inclusions in PEDV co-infections was highly variable. In Chlamydia abortus co-infection experiments, three types of inclusions were observed: (i) small

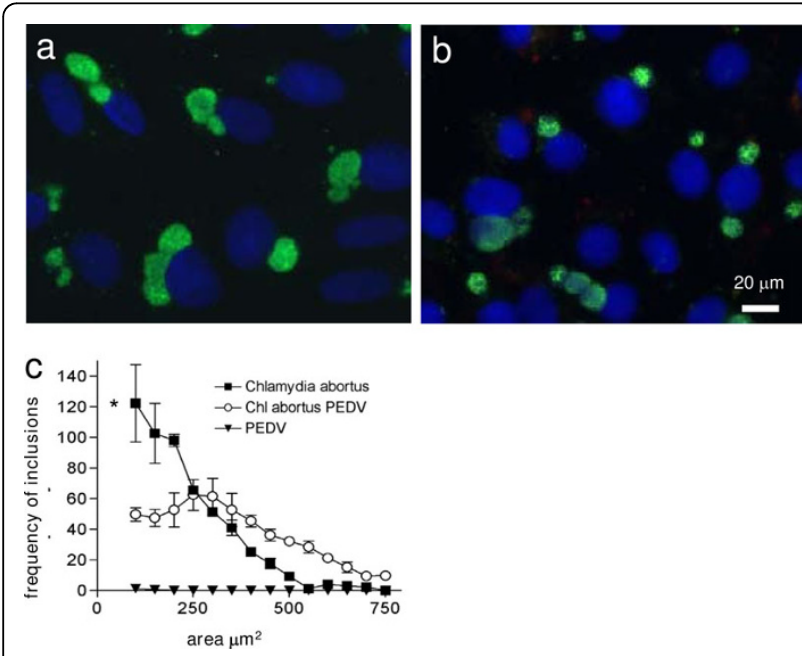

Figure 2 Morphology of Chlamydia abortus mono- and coinfection with PEDV. a) Vero cells were infected with Chlamydia abortus $1 \mathrm{MO}$ for $39 \mathrm{~h}$ stained with an anti-Chlamydia antibody (green). Nuclei of Vero cells are visualized by DAPI stain (blue); b) Vero cells were infected with Chlamydia abortus with subsequent PEDV inoculation and stained as with an anti-Chlamydia antibody and DAPI; c) Frequency of inclusions with various sizes was calculated and mono and double infected cells were compared according to the inclusion size. The difference between mono and double infected monolayers was statistically analyzed using students t-test. The groups were significantly different with $p=0.0132$.

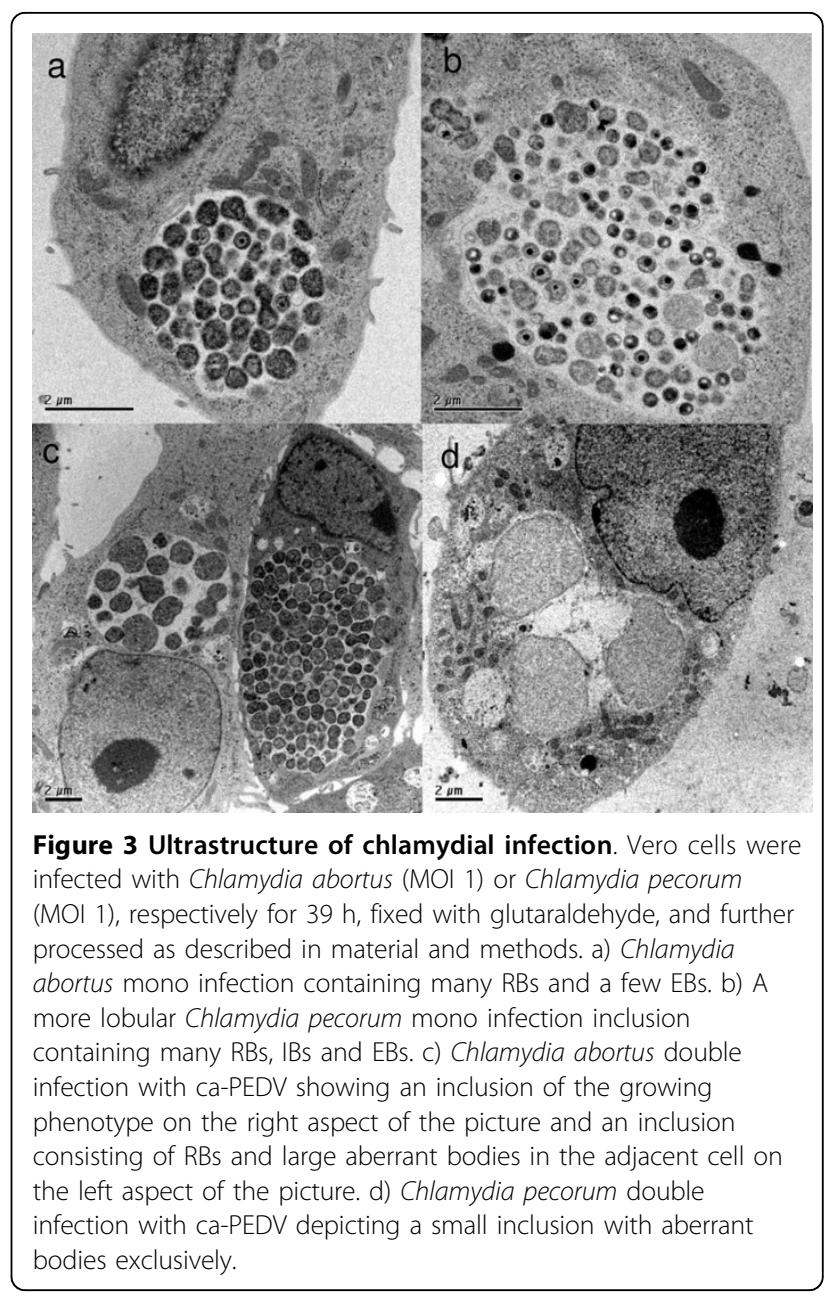

inclusions consisting of 1-10 aberrant bodies (ABs), (ii) medium-sized inclusions consisting of $\mathrm{ABs}$ and reticulate bodies (RBs), and (iii) large (normal) inclusions consisting of EBs as seen in the single infection experiments (Figure 2b).

In contrast, dual infections with ca-PEDV and Chlamydia pecorum resulted in the exclusive production of aberrant inclusions containing between 2-50 ABs. Chlamydial inclusions in viral syncytia grew even larger than in non-viral infected Vero cells. Overall, no normal chlamydial inclusions were observed (Figure 1a \&1b).

Image analysis was used to compare inclusion size in single chlamydiae-infected Vero cells with the inclusion size in Vero monolayers that subsequently underwent ca-PEDV virus infection. To this end, inclusion size was determined in $\mu \mathrm{m}^{2}$ and all inclusions were assembled into groups covering $50 \mu^{2}$ and multiples of this area.

The average frequency of Chlamydia pecorum inclusions between $100 \mu \mathrm{m}^{2}$ and $400 \mu \mathrm{m}^{2}$ was significantly reduced when cells were subsequently infected with caPEDV. In other words, Chlamydia pecorum inclusions 
were highly significant smaller in ca-PEDV dual infections than in those infections without the addition of virus (Figure 1d) as analyzed by $\mathrm{t}$-test $(\mathrm{p}=0.0044)$. The additional changes observed in the shape of all inclusions growing in virus-infected monolayers indicated the induction of Chlamydia pecorum persistence, since the finely dispersed staining reverted to grape-like structures (Figure 1a \&1b).

The changes of chlamydial inclusion size by subsequent virus addition to Chlamydia abortus are different to those we observed in the Chlamydia pecorum dual infection experiments. The frequency of inclusions observed between a size range of $0-200 \mu \mathrm{m}^{2}$ was significantly ( $\mathrm{p}=0.0132)$ reduced under virus infection but the amount of medium sized and big inclusions 300 $700 \mu \mathrm{m}^{2}$ was increased (Figure 2c). The morphology of Chlamydia abortus inclusions was also found to differ in the population when co-infected with ca-PEDV. Smaller inclusions were generally observed in aberrant shapes compared to larger inclusions, which appeared similar to normal actively growing inclusions showing finely dispersed staining (Figure 2b). This effect might be due to an incomplete induction of persistence of Chlamydia abortus when cells were ca-PEDV coinfected.

\section{Co-infection with ca-PEDV induced ultrastructural morphological changes in Chlamydia abortus and Chlamydia pecorum}

Persistent forms of Chlamydia trachomatis and Chlamydia pneumoniae are well described by their characteristic electron microscopic appearance $[2,13,14]$. Thus, chlamydial ultrastructure in single and co-infected cells was compared by transmission electron microscopy (TEM).

At $24 \mathrm{~h}$ after viral infection, viral-induced syncytia containing vacuoles filled with viral particles were present in ca-PEDV-monoinfected and dual infections. The viral particles showed the typical Coronavirus morphology with a diameter between 50 to $130 \mathrm{~nm}$ (data not shown). At $39 \mathrm{~h}$ after chlamydial infection, large intracytoplasmic chlamydial inclusions in single infected cells could be observed in Vero cells infected with Chlamydia abortus or Chlamydia pecorum. The inclusions observed contained variable numbers of morphologically normal RBs and EBs and were generally located near the host cell nucleus, often surrounded by mitochondria (Figure $3 \mathrm{a}$ and $3 \mathrm{~b}$ ).

TEM examinations of mixed infections (ca-PEDV and Chlamydia abortus or Chlamydia pecorum) revealed aberrant chlamydial inclusions containing fewer bacteria than typical inclusions and were located in viral syncytia or single cells without viral infection. Aberrant inclusions consisted of reticulate-like, pleomorphic, aberrant bodies (ABs), which were in general larger in diameter (up to $2 \mu \mathrm{m}$ ) than typical reticulate bodies (RBs), with a sparse densitometric appearance and no re-differentiation into elementary bodies (EBs). As already observed in IF investigations, three types of inclusions were present in dual infections with ca-PEDV and Chlamydia abortus (Figure 3c), whereas dual infections with caPEDV and Chlamydia pecorum resulted in the exclusive production of aberrant inclusions consisting of 2-50 ABs (Figure 3d).

Neither chlamydial inclusions nor ca-PEDV virions were visible in mock-infected cells.

\section{ca-PEDV superinfection inhibition of infectious chlamydial} EBs is chlamydial strain-specific

Previous studies have demonstrated that chlamydial persistent forms are non-infectious [2]. Reduced number or even a lack of EBs in co-infected cells in TEM suggested arrested chlamydial developmental cycle with halted maturation from RB to EB. To ascertain the effect of caPEDV inhibition of chlamydial EB production, the yield of infective chlamydial progeny was determined after 40 $h$ of re-infection in three independent experiments for Chlamydia abortus (Figure 4a) and for Chlamydia pecorum (Figure $4 \mathrm{~b}$ ). Neither mock nor ca-PEDV monoinfected cells produced detectable infectious EBs, whereas Chlamydia abortus and Chlamydia pecorum single infections cells produced abundant EBs. Coinfected cells produced fewer infectious EBs than nonviral infected cells, demonstrating that production of infectious chlamydial progeny was essentially diminished by ca-PEDV-co-infection. Eradication of infectious EB production was almost complete in Chlamydia pecorum double infection, analyzed by reinfection experiments and found to be statistically different as analyzed by $\mathrm{t}$ test $(\mathrm{p}=0.0145)$ (Figure $4 \mathrm{~b})$. In Chlamydia abortus reinfection analysis, several EBs could still be observed in spite of the co-infection with ca-PEDV (Figure 4a). Statistical analysis by t-test revealed no statistical

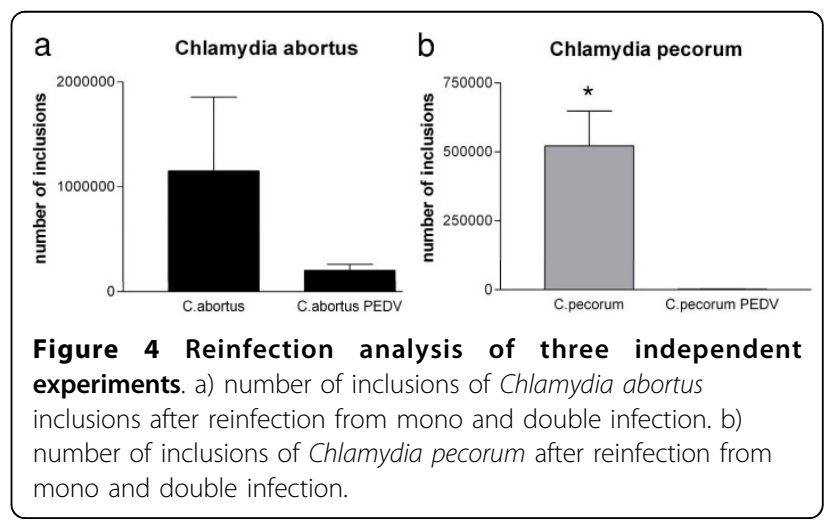


difference $(\mathrm{p}=0.2523)$ presumably due to the high variation in the data.

This data is consistent with the observations from our IF and ultrastructural analysis.

\section{Chlamydial co-infection does alter ca-PEDV infection depending on the chlamydial species but does not alter viral ultrastructure}

To determine whether chlamydial pre-infection altered subsequent viral infection, numbers of syncytia and caPEDV-infected cells from single and co-infected monolayers of three unrelated experiments were counted. Mock-infected and Chlamydia only infected cells produced no virions. The difference between virus-infected cells and co-infection with Chlamydia abortus was minimal. The number of syncytia detected were within the same range (data not shown) indicating that chlamydial co-infection with Chlamydia abortus does not alter caPEDV infection or the development of syncytia. In contrast, numbers of syncytia in co-infection with Chlamydia pecorum were reduced compared to single ca-PEDV infection (Table 1).

Overall numbers of single viral infected cells were low in both single and co-infection experiments, and no significant difference between the two chlamydial species was obvious (data not shown).

Viral morphology was also studied by TEM. In caPEDV single and co-infected cells, viral particles were unaltered indicating that chlamydial co-infection did not induce any changes in viral ultrastructural morphology.

\section{Discussion}

While a previous study [12] primarily investigated the interaction of ca-PEDV and Chlamydiaceae in mixed infections to detect possible synergistic or additive effects of these two pathogens, questions remained about whether viral infection could potentially induce the persistent chlamydial phenotype. Enlarged chlamydial inclusions were described in that study in the caPEDV co-infection model with Chlamydia abortus and Chlamydia pecorum but no further ultrastructural analysis has been subsequently performed. In this study, in vitro models of Chlamydia abortus and Chlamydia pecorum persistence were established using co-infection with ca-PEDV. Several experimental methods were used to demonstrate the characteristic features of chlamydial persistence, including altered ultrastructural morphology and decreased production of infectious EBs. Our results demonstrated that ca-PEDV-co-infection alters the chlamydial developmental cycle similarly to other inducers of chlamydial persistence. A similar co-infection model has been recently described by Deka et al. (2006) [15]. In that study, it was shown that Chlamydia trachomatis enters a viable but non-cultivable, persistent state with herpes simplex virus type 2 (HSV-2) co-infected host cells. In contrast, a similar study investigating a coinfection model with Chlamydia trachomatis and genital mycoplasmas, Mycoplasma genitalium and Mycoplasma hominis, did not change the morphology of chlamydial RBs, indicating that co-infection of these two microorganisms is likely to be independent and not related to the onset of chlamydial persistence [16]. In the study by Deka et al. (2006) [15], HeLa monolayers were first infected with Chlamydia trachomatis and $24 \mathrm{~h}$ later with HSV-2. In our study, the optimal experimental protocol for co-infection procedure was developed, based on our own earlier study [12], and optimization experiments performed as a part of the current study (data not shown). To this end, Vero monolayers were first infected with Chlamydia and later with ca-PEDV, thus the suspected inducer of persistence would be introduced after chlamydial infection and differentiation into RBs. Simultaneous infection of Chlamydia and ca-PEDV has been performed earlier [12], but did not result in persistent infection in our preliminary experiments (data not shown) and was not considered further as interference of chlamydial infection and concurrent viral uptake could have influenced the results. Viral infection and subsequent development of syncytia was not affected by co-infection with Chlamydia abortus as demonstrated by unaltered numbers of syncytia observed in the coinfection experiments. In contrast, viral syncytia formation was dramatically decreased in Vero cells double infected with ca-PEDV and Chlamydia pecorum. If Chlamydia pecorum infection might induce a down regulation of the host PEDV receptor needed for syncytium formation at 14-15 hours post-chlamydial infection, this could produce a reduction in syncytium formation without reducing viral entry or replication - the possible persistence inducer mechanism.

Interestingly, chlamydial persistence was more prominent in co-infection with Chlamydia pecorum than with Chlamydia abortus, indicating possible species-specific differences. Limited reports are available for in vitro models of chlamydial persistence from non-Chlamydia trachomatis and Chlamydia pneumoniae strains. Kaltenboeck and Storz (1992) [17] suggested that strain 1710S of Chlamydia pecorum is highly nutrient dependent and this could elicit aberrant forms. Indeed, aberrant forms of this strain were significantly present in our study. Previously, only limited data have been published on persistent infection of L cells with an ovine abortion strain of Chlamydia psittaci (current classification: Chlamydia abortus) [18]. It should be noted, that in the latter study, chlamydial persistence was not demonstrated using the characteristic features now associated with the morphology of persistent chlamydial infections. Detailed description of electron microscopic 
observations on the effects of penicillin on the morphology of Chlamydia psittaci Cal10 in L cells showing aberrant chlamydial stages were published by Matsumoto and Manire [13].

The different occurence of persistent forms in coinfection with Chlamydia abortus and Chlamydia pecorum, respectively, has not been described before. Differences between persistence behaviour are already known (reviewed by Hogan et al., 2004) [1] not only between different chlamydial species but also between different serovars and strains of Chlamydia pneumoniae and Chlamydia trachomatis, respectively. The fact that Chlamydia pecorum strain $1710 \mathrm{~S}$ is an original swine isolate whereas Chlamydia abortus strain S26/3 originates from a sheep abortion and, thus, from another animal species could have an impact but needs further investigation.

The mechanism by which ca-PEDV interferes with chlamydial developmental cycle and chlamydial persistence is still unclear. It is known that Vero cells, a monkey kidney epithelial cell line, is deficient for Interferon production [19]; thus, this cytokine group well known to be capable of inducing in vitro persistence in Chlamydia pneumoniae [1], cannot be relevant for our co-infection persistence model. Co-infection experiments with caPEDV are best performed with Vero cells, as they have been shown to be permissive for viral replication in contrast to other cell lines such as PD5, PK 15, and HRT18 cell lines [9]. Specific measurements of primate cytokines in our co-infection model are planned in the future to elucidate the mechanism leading to chlamydial persistence. The Herpes simplex virus (HSV) co-induced Chlamydia trachomatis persistence model [15] has been recently been shown not to be mediated by any known persistence inducer or anti-chlamydial pathway recently $[20,21]$. Instead, it was hypothesized by the authors that HSV-2 attachment and/or entry into the host cell is sufficient for stimulating chlamydial persistence, suggesting a potential novel host signaling pathway could be responsible for inducing chlamydial persistence. A very recent publication by the same group showed that HSV replication is not necessary for persistence induction and that chlamydial activity could be recovered after coinfection with UV-inactivated HSV-2. Finally, it was concluded that the interaction of HSV glycoprotein D with the host cell surface is crucial to trigger chlamydial persistence [22].

Female genital tract infection often has a complex etiology, where Chlamydia trachomatis is present together with one or more genital agents. Epidemiological and clinical studies have shown that double infection with HSV-2 and Chlamydia trachomatis occurs in vivo; thus, the in vitro model described by Deka et al. (2006) [15] represents a realistic situation in human medicine. Similarities exist to the in vitro model established in this study as simultaneous intestinal infection with different pathogens is possible in swine in vivo. A recent study [23] documented the occurrence of aberrant chlamydial bodies in vivo in intestinal tissues of pigs. In this study, aberrant bodies of Chlamydia suis were demonstrated and characterized in the gut of pigs experimentally infected with Salmonella typhimurium by transmission electron microscopy. It was concluded by Pospischil et al. [23] that aberrant bodies occur in vivo in pigs and that the gnotobiotic pig model might be suitable for the study of chlamydial persistence in vivo.

Available intestinal tissues from experimentally infected gnotobiotic piglets (single infection and coinfection with Chlamydia and ca-PEDV, respectively) will be investigated in the future with the aim of further characterization of $\mathrm{ABs}$ in vivo. Although chronic chlamydial diseases in animals are of economic impact, the pig model may also reveal the important link between persistence in vitro and in vivo and, thus, help to elucidate mechanisms of chronic human chlamydial infections in the future.

\section{Conclusions}

The present study reports a new persistence model of Chlamydia in co-infection with porcine epidemic diarrhea virus (PEDV). PEDV-co-infection altered the chlamydial developmental cycle similarly to other known inducers of chlamydial persistence. This new animal model could provide the important link between persistence in vitro and in vivo and, thus, would help to elucidate mechanisms of chronic human chlamydial infections in the future.

\section{Methods}

\section{Media and cells}

Growth medium (GM) for normal cell propagation was Minimal Essential Medium (MEM) with Earle's salts, 25 mM HEPES, without L-Glutamine (GIBCO, Invitrogen, Carlsbad, CA) and supplemented with $10 \%$ fetal calf serum (FCS) (BioConcept, Allschwil, Switzerland), 4 $\mathrm{mM}$ GlutaMAX-I (200 mM, GIBCO) and $0.2 \mathrm{mg} / \mathrm{ml}$ gentamycin (50 mg/ml, GIBCO).

GM without gentamycin was used for the propagation of cells for infection experiments. Infection medium was prepared as GM but without gentamycin and FCS, and was used for the infection and for the $24 \mathrm{~h}$ incubation period after the infection with ca-PEDV, respectively. Incubation medium was prepared as GM without gentamycin, freshly supplemented with $1 \mu \mathrm{g} / \mathrm{ml}$ cycloheximide (Sigma, Buchs SG, Switzerland), and used after an infection for estimation of the chlamydial titer (IFU determination). 
Vero 76 cells (African green monkey kidney cells, CRL 1587 American Type Culture Collection) were seeded on round plastic coverslips (13 $\mathrm{mm}$ diameter, Bibby Sterilin, Stone, UK) and cultured in GM without gentamycin at $37^{\circ} \mathrm{C}$ until they reached confluence. Before inoculation, the cells were washed once with phosphate buffered saline (PBS).

\section{Chlamydial strains}

Two different chlamydial strains of Chlamydiaceae were used in this study: Chlamydia abortus S26/3 (ovine abortion strain, kindly donated by Dr. G.E. Jones, Moredun Research Institute, Edinburgh, GB) and Chlamydia pecorum $1710 \mathrm{~S}$ (intestinal swine isolate, kindly provided by Prof. J. Storz, Baton Rouge, Louisiana, LA, USA). For initial culturing, chlamydial strains were cultured in embryonated chicken eggs, and yolk sac material was harvested, diluted 1:2 in sucrose-phosphate-glutamate (SPG) medium and stored at $-80^{\circ} \mathrm{C}$. Yolk sac-derived chlamydiae were then propagated in HEp-2 cell (ATCC CCL-23) monolayers and elementary bodies (EBs) were harvested and purified by disruption of HEp- 2 cell monolayers with a cell scraper, sonication and centrifugation over a renografin density gradient as described elsewhere [24]. EB suspensions were stored in sucrosephosphate-glutamic acid buffer at $-80^{\circ} \mathrm{C}$, after which viable titers were established using standard methods. MOI of 1 was used for chlamydial monoinfection and mixed infection, respectively.

\section{PEDV}

Ca-PEDV strain CV777 (kindly provided by Prof. Dr. M. Ackermann, Institute of Virology, University of Zurich) was propagated as previously described [9]. The virus $\left(10^{5,5} \mathrm{TCID}_{50} / \mathrm{ml}\right)$ was used undiluted $\left(1 \mathrm{ml} 10^{5,5}\right.$ $\left.\mathrm{TCID}_{50} / \mathrm{ml}\right)$.

\section{Co-infection experimental design}

Vero cells, an African green monkey kidney cell line (ATCC CRL 1587), were used for all infection experiments. They were propagated in GM without gentamycin at $37^{\circ} \mathrm{C}$ in an atmosphere of $5 \% \mathrm{CO}_{2}$. Vero cells were divided into four groups: for mock infection, chlamydial infection, ca-PEDV infection, and both Chlamydia and ca-PEDV double infection. Host cells were infected with a MOI of 1 for Chlamydia and an infective dose of $1 \times 10^{5,5} \mathrm{TCID}_{50} / \mathrm{ml}$ for ca-PEDV, respectively. For ca-PEDV monoinfections and negative controls, infection medium was used.

All co-infection experiments were done three times and monoinfections with Chlamydia and ca-PEDV were performed simultaneously. The optimal experimental protocol (adding the virus several hours after chlamydial infection) for co-infection procedure was developed before (data not shown).

For dual infections, cell monolayers were first infected with Chlamydia at a MOI of 1 . All coverslips were centrifuged at $1000 \times \mathrm{g}$ for $1 \mathrm{~h}$ at $25^{\circ} \mathrm{C}$. Timepoint $0\left(\mathrm{~T}_{0}\right)$ was defined after centrifugation and supernatant was replaced subsequently by incubation medium. Infected monolayers were then incubated for $14 \mathrm{~h}$ at $37^{\circ} \mathrm{C}\left(\mathrm{T}_{0}\right.$ $\mathrm{T}_{14}$ ). All cell layers for dual infections or ca-PEDV monoinfection were exposed to a ca-PEDV suspension $\left(1 \times 10^{5,5} \mathrm{TCID}_{50}\right)$, the samples were centrifuged again for $1000 \times \mathrm{g}$ for $1 \mathrm{~h}$ at $25^{\circ} \mathrm{C}$ and incubated for $24 \mathrm{~h}$ at $37^{\circ} \mathrm{C}$. After this incubation period, all monolayers were fixed and further investigated by indirect immunofluorescence and transmission electron microscopy. Re-infection experiments were performed to compare the production of infectious chlamydial elementary bodies (EBs) between monoinfections and mixed infections.

\section{Indirect Immunofluorescence}

For indirect immunofluorescence analyses, infected cells were fixed in absolute methanol $\left(-20^{\circ} \mathrm{C}\right)$ for $10 \mathrm{~min}$. and IF labeling of cell cultures was performed immediately after fixation. For viral antigen detection, a mouse monoclonal antibody against the $M$ protein of PEDV (mcAb 204, kindly provided by Prof. Dr. M. Ackermann, Institute of Virology, University of Zurich), diluted 1:4 in PBS supplemented with BSA, and an Alexa Fluor 594-conjugated secondary antibody (goat anti-mouse, 1:500, Molecular Probes, Eugene, USA) were used. Chlamydial inclusions were labeled with a Chlamydiaceae family-specific mouse monoclonal antibody directed against the chlamydial lipopolysaccharide (mLPS; Clone ACI-P, Progen, Heidelberg, Germany) and a secondary Alexa Fluor 488-conjugated secondary antibody (goat anti-mouse, 1:500, Molecular Probes). DNA was labeled with $1 \mu \mathrm{g} / \mathrm{ml} \mathrm{4',6-Diamidin-2'-phenylindoldihydro-}$ chlorid (DAPI, Molecular Probes). All staining procedures were conducted at room temperature. Antibody incubations were carried out for $1 \mathrm{~h}$ (primary antibodies) or $45 \mathrm{~min}$ (secondary antibodies), respectively, with three washes of PBS following fixation, between and after applications of the different antibodies. Dually infected cell layers were stained using sequential double immunofluorescence labeling. Uninfected Vero cells were used as a negative control. Coverslips were mounted with Immumount (Shandon, Pittsburgh, USA) on glass slides and investigated using a Leica fluorescence microscope.

\section{Transmission electron microscopy}

Coverslips from all experimental conditions were fixed in $2.5 \%$ glutaraldehyde (Electron Microscopy Sciences, 
Ft. Washington, USA) for 1-2 $\mathrm{h}$, and processed by routine methods for embedding in epoxy resin (Fluka). Appropriate areas for ultrastructural investigation were selected using semithin sections $(1 \mu \mathrm{m})$ stained with toluidine blue (Fluka, Buchs SG, Switzerland). Ultrathin sections $(80 \mathrm{~nm})$ were mounted on gold grids (Merck Eurolab AG, Dietlikon, Switzerland), contrasted with uranyl acetate dihydrate (Fluka) and lead citrate (lead nitrate and tri-natrium dihydrate; Merck Eurolab AG) and investigated in a Philips CM10 electron microscope.

\section{Chlamydial titration by subpassage}

At $39 \mathrm{~h}$ after chlamydial infection, monolayers were scraped into $1 \mathrm{ml}$ of cold infection medium, pelleted and resuspended in $1 \mathrm{ml}$ of fresh medium. Infected host cells were lysed by sonication and centrifuged $(500 \mathrm{~g}$ for $5 \mathrm{~min}$ ) to remove pellet cell debris. Supernatants were centrifuged once $(4,000 \mathrm{~g}$ for $60 \mathrm{~min})$. Final EB pellets were resuspended in $200 \mu \mathrm{l}$ of SPG and used to infect Vero cells plated on glass coverslips in duplicate in dilution series. All coverslips were centrifuged at $1000 \times \mathrm{g}$ for $1 \mathrm{~h}$ at $25^{\circ} \mathrm{C}$. After centrifugation, the Vero cells were refed with medium containing $1 \mu \mathrm{g} / \mathrm{ml}$ cycloheximide and subsequently incubated for $40 \mathrm{~h}$ at $37^{\circ} \mathrm{C}$. Fixation and staining of Chlamydia, ca-PEDV and DNA was performed as described above. The number of inclusions in 20 random microscopic fields per sample was determined using a Leica fluorescence microscope at a magnification of $200 \times$. Duplicate coverslips were counted and the counts were averaged. The number of inclusion-forming units (IFU) in the indiluted inoculum was then calculated and expressed as IFU per $10^{6}$ cells as described by Deka et al., 2006 [15].

\section{Imaging and statistical analyses}

From duplicate samples of three independent experiments uniform random sampled images were acquired using a widefield microscope (Leica LX, Leica Microsystems Mannheim, Germany). Cells and inclusions were automatically detected according to size, shape and intensity and counted using Imaris (Bitplane AG, Zürich Switzerland).

\footnotetext{
Acknowledgements

The authors would like to thank Lisbeth Nufer of the laboratory staff at the Institute of Veterinary Pathology, Zurich, for her excellent technical assistance. We would also like to thank Dr. Monika Engels and Eva Loepfe, Institute of Virology (Head: Prof. M. Ackermann), Vetsuisse Faculty, University of Zurich for providing the porcine epidemic diarrhea virus. We thank Dr. Adam Polkinghorne, Queensland University of Technology, Brisbane, Australia, for manuscript editing.

This work was supported by a grant from the University of Zurich (Forschungskredit)
}

\section{Author details}

${ }^{1}$ Institute of Veterinary Pathology, Vetsuisse Faculty, University of Zurich Zurich, Switzerland. ${ }^{2}$ Center for Microscopy and Image Analysis, University of Zurich, Zurich, Switzerland.

\section{Authors' contributions}

NB conceived of the study, planned the experiments, and drafted the manuscript. CD and UZ performed the imaging and statistical analyses. AS and CK carried out the cell culture experiments including

immunofluorescence and transmission electron microscopy. AP participated in the design and coordination of the study and helped to draft the manuscript. All authors read and approved the final manuscript.

\section{Competing interests}

The authors declare that they have no competing interests.

Received: 13 April 2010 Accepted: 27 July 2010 Published: 27 July 2010

\section{References}

1. Hogan RJ, Mathews SA, Mukhopadhyay S, Summersgill JT, Timms P: Chlamydial persistence: beyond the biphasic paradigm. Infect Immun 2004, 72:1843-1855.

2. Beatty $\mathrm{WL}$, Morrison RP, Byrne Gl: Persistent chlamydiae: from cell culture to a paradigm for chlamydial pathogenesis. Microbiol Rev 1993, 58:686-699.

3. Beatty WL, Byrne Gl, Morrison RP: Morphologic and antigenic characterization of interferon gamma-mediated persistent Chlamydia trachomatis infection in vitro. Proc Natl Acad Sci USA 2003, 90:3998-4002.

4. Taylor DJ: Chlamydiae. Diseases of Swine lowa State University Press, Ames, lowaStraw BE, Allaire SD, Mengeling WL, Taylor DJ , 8 1999, 619-624.

5. Nietfeld JC, Leslie-Steen $P$, Zeman DH, Nelson D: Prevalence of intestinal chlamydial infection in pigs in the midwest, as determined by immunoperoxidase staining. Am J Vet Res 1997, 58:260-264.

6. Szeredi L, Schiller I, Sydler T, Guscetti F, Heinen E, Corboz L, Eggenberger E, Jones GE, Pospischil A: Intestinal Chlamydia in finishing pigs. Vet Pathol 1996, 33:369-374.

7. Pospischil A, Wood RL: Intestinal Chlamydia in pigs. Vet Pathol 1987, 24:568-570.

8. Pensaert MB, Debouck P: A new coronavirus-like particle associated with diarrhea in swine. Arch Virol 1978, 58:243-247.

9. Hofmann M, Wyler R: Propagation of the virus of porcine epidemic diarrhea in cell culture. J Clin Microbiol 1988, 26:2235-2239.

10. Duarte M, Tobler K, Bridgen A, Rasschaert D, Ackermann M, Laude H: Sequence analysis of the porcine epidemic diarrhea virus genome between the nucleocapsid and spike protein genes reveals a polymorphic ORF. Virology 1994, 198:466-476.

11. Tobler K, Ackermann M: PEDV leader sequence and junction sites. Corona and related viruses Plenum Press, New YorkTalbot PJ, Levy GA 1994, 541-542.

12. Stuedli A, Grest P, Schiller I, Pospischil A: Mixed infections in vitro with different Chlamydiaceae strains and a cell culture adapted porcine epidemic diarrhea virus. Vet Microbio/ 2005, 106:209-223.

13. Matsumoto A, Manire GP: Electron microscopic observations on the effects of penicillin on the morphology of Chlamydia psittaci. J Bacteriol 1970, 101:278-285.

14. Byrne Gl, Ouellette SP, Wang Z, Rao JP, Lu L, Beatty WL, Hudson AP: Chlamydia pneumoniae expresses genes required for DNA replication but not cytokinesis during persistent infection of HEp-2 cells. Infect Immun 2001, 69:5423-9.

15. Deka S, Vanover J, Dessus-Babus S, Whittimore J, Howett MK, Wyrick PB, Schoborg RV: Chlamydia trachomatis enters a viable but non-cultivable (persistent) state within herpes simplex virus type 2 (HSV-2) co-infected host cells. Cell Microbiol 2006, 8:149-162.

16. Baczynska A, Birkelund S, Christiansen G: Chlamydia trachomatis and genital mycoplasmas in the co-infection model - in vitro study. Proceedings of the Eleventh International Symposium on Human Chlamydial Infections: 18-23 June 2006; Niagara-on-the-Lake, Ontario, Canada International Chlamydia Symposium, San Francisco, CAChernesky M, Caldwell H, Christiansen G, Clarke IN, Kaltenboeck B, Knirsch C, Kuo CC, Mahony J, Rank RG, Saikku P, Schachter J, Stamm WE, Stephens RS, Summersgill JT, Timms P, Wyrick PB 2006, 225-228.

17. Kaltenboeck B, Storz J: Biological properties and genetic analysis of the omp A locus in chlamydiae isolated from swine. Am J Vet Res 1992, 53:1482-1487.

18. Perez-Martinez JA, Storz J: Persistent infection of $L$ cells with an ovine abortion strain of Chlamydia psittaci. Infect Immun 1985, 50:453-8. 
19. Chew T, Noyce R, Collins SE, Hancock MH, Mossman KL: Characterization of the interferon regulatory factor 3-mediated antiviral response in a cell line deficient for IFN production. Mol Immunol 2009, 46:393-9, 2009.

20. Deka S, Vanover J, Sun J, Kintner J, Whittimore J, Schoborg RV: An early event in the herpes simplex virus type- 2 replication cycle is sufficient to induce Chlamydia trachomatis persistence. Cell Microbiol 2007, 9:725-37.

21. Vanover J, Sun J, Deka S, Kintner J, Duffourc MM, Schoborg RV: Herpes simplex virus co-infection-induced Chlamydia trachomatis persistence is not mediated by any known persistence inducer or anti-chlamydial pathway. Microbiology 2008, 154:971-8.

22. Vanover J, Kintner J, Whittimore J, Schoborg RV: Interaction of HSV-2 glycoprotein D with the host cell surface is sufficient to induce Chlamydia trachomatis persistence. Microbiology 2010.

23. Pospischil A, Borel N, Chowdhury EH, Guscetti F: Aberrant chlamydial developmental stages in the gastrointestinal tract of pigs spontaneously and experimentally infected with Chlamydia suis. Vet Microbiol 2009, 135:147-56.

24. Howard L, Orenstein NS, King NW: Purification on renografin density gradients of Chlamydia trachomatis grown in the yolk sac of eggs. Appl Microbiol 1974, 27:102-106.

doi:10.1186/1471-2180-10-201

Cite this article as: Borel et al:: Mixed infections with Chlamydia and porcine epidemic diarrhea virus - a new in vitro model of chlamydial persistence. BMC Microbiology 2010 10:201.

\section{Submit your next manuscript to BioMed Central and take full advantage of:}

- Convenient online submission

- Thorough peer review

- No space constraints or color figure charges

- Immediate publication on acceptance

- Inclusion in PubMed, CAS, Scopus and Google Scholar

- Research which is freely available for redistribution

Submit your manuscript at www.biomedcentral.com/submit 